\title{
Adaptive algorithm for solving the SCFPP of demicontractive operators without a priori knowledge of operator norms
}

\author{
Duangkamon Kitkuan, Poom Kumam, Vasile Berinde and \\ Anantachai Padcharoen
}

\begin{abstract}
In this paper, we study the split common fixed point problem in Hilbert spaces. We find a common solution of the split common fixed point problem for two demicontractive operators without a priori knowledge of operator norms. A strong convergence theorem is obtained under some additional conditions and numerical examples are included to illustrate the applications in signal compressed sensing and image restoration.
\end{abstract}

\section{Introduction}

Let $H$ be a real Hilbert space. The convex feasibility problem (shortly, (CFP)) is defined as follows:

$$
\text { find } x^{*} \in H \text { such that } x^{*} \in \bigcap_{i=1}^{m} C_{i} \text {, }
$$

where $m \geq 1$ is an integer and each $C_{i}$ is a nonempty closed convex subset of $H$.

Key Words: common solution, demicontractive operator, split feasibility problem, split common fixed point problem, Hilbert space.

2010 Mathematics Subject Classification: Primary 47H09 ; Secondary 49M05.

Received: 12.12 .2018

Accepted: 31.01.2019 
A special case of the problem (CFP) is the following split feasibility problem (shortly, (SFP)):

$$
\text { find } x^{*} \in C \text { such that } A x^{*} \in Q \text {, }
$$

where $C$ and $Q$ are two closed convex subsets of two Hilbert spaces $H_{1}$ and $H_{2}$, respectively, and $A: H_{1} \rightarrow H_{2}$ is a bounded linear operator.

Byrne [2] introduced a very popular algorithm $\left\{x_{n}\right\}$ that solves the problem (SFP):

$$
x_{n+1}=P_{C}\left(x_{n}-\gamma A^{*}\left(I-P_{Q}\right) A x_{n}\right)
$$

for each $n \geq 0$, where $P_{C}$ and $P_{Q}$ are metric projections onto $C$ and $Q$, respectively, $A^{*}$ denotes the adjoint of the mapping $A: H_{1} \rightarrow H_{2}$ and $\gamma \in$ $\left(0, \frac{2}{\lambda}\right)$ with $\lambda$ being the spectral radius of the mapping $A^{*} A$.

If $C=F(T)$ and $Q=F(S)$, then, from the problem (SFP), we have the split common fixed point problem (shortly, (SCFPP)) which is defined as follows:

$$
\text { find a point } x^{*} \in F(T) \text { such that } A x^{*} \in F(S) \text {, }
$$

where $F(T), F(S)$ stand for the fixed point sets of the mappings $T: H_{1} \rightarrow H_{1}$, $S: H_{2} \rightarrow H_{2}$, respectively, and $A: H_{1} \rightarrow H_{2}$ is a bounded linear operator. We denote the set of solutions of the problem (SCFPP) by

$$
\Gamma:=\left\{y^{*} \in C: A y^{*} \in Q\right\}=C \cap A^{-1}(Q) .
$$

Let $T: H_{1} \rightarrow H_{1}$ and $S: H_{2} \rightarrow H_{2}$ be two mappings such that

$$
C:=F(T)=\left\{x^{*} \in H_{1}: T x^{*}=x^{*}\right\} \neq \emptyset
$$

and

$$
Q:=F(S)=\left\{x^{*} \in H_{2}: S x^{*}=x^{*}\right\} \neq \emptyset .
$$

In this paper, we prove a result on the existence of solutions of the split common fixed point problem (SCFPP) for two demicontractive mappings $T: H_{1} \rightarrow H_{1}$ and $S: H_{2} \rightarrow H_{2}$ with $C:=F(T) \neq \emptyset$ and $Q:=F(S) \neq \emptyset$ and obtain the solution by a new algorithm $\left\{x_{n}\right\}$.

Censor and Segal [3] introduced, in finite-dimensional spaces, the following algorithm $\left\{x_{n}\right\}$ for solving the problem (SCFPP):

$$
x_{n+1}=T\left(x_{n}+\tau A^{t}(S-I) A x_{n}\right)
$$

for each $n \geq 1$, where $\tau \in\left(0, \frac{2}{\gamma}\right)$ with $\gamma$ being the largest eigenvalue of the matrix $A^{t} A$ ( $A^{t}$ is matrix transposition). 
Moudafi [18] proved some weak convergence theorems in Hilbert spaces when two mappings $T$ and $S$ are quasi-nonexpansive mappings by using the following relaxed algorithm $\left\{x_{n}\right\}$ :

$$
\left\{\begin{array}{l}
y_{n}=x_{n}+\tau A^{*}(S-I) A x_{n}, \\
x_{n+1}=\left(1-\alpha_{n}\right) y_{n}+\alpha_{n} T y_{n}
\end{array}\right.
$$

for each $n \geq 1$, where $\alpha_{n} \in(0,1)$ and $\tau \in\left(0, \frac{1}{\beta \gamma}\right)$ with $\gamma$ being the spectral radius of the operator $A^{*} A$ and $\beta \in(0,1)$.

Moudafi [17] also proposed an iterative algorithm $\left\{x_{n}\right\}$ to solve the problem (SCFPP), where $S$ and $T$ are demicontractive mappings as follows:

$$
\left\{\begin{array}{l}
u_{n}=x_{n}+\tau A^{*}(S-I) A x_{n}, \\
x_{n+1}=\left(1-\alpha_{n}\right) u_{n}+\alpha_{n} T u_{n}
\end{array}\right.
$$

for each $n \geq 1$, where $\alpha_{n} \in(0,1)$ and $\tau \in\left(0, \frac{1-\mu}{\gamma}\right)$ with $\gamma$ being the spectral radius of the operator $A^{*} A$ and $\beta \in(0,1)$.

In particular, it was noted that the problem (SCFPP) is equivalent to solving the following fixed point problem

$$
x=x-\tau\left((x-T x)+A^{*}(I-S) A\right) x,
$$

where $\tau>0$ is a constant and $T$ and $S$ are directed operators.

Based on the fixed point equation approach, Wang [24] suggested the following algorithm $\left\{x_{n}\right\}$ :

$$
x_{n+1}=x_{n}-\tau\left(\left(x_{n}-T x_{n}\right)+A^{*}(I-S) A x_{n}\right),
$$

where $T: \mathbb{R}^{n} \rightarrow \mathbb{R}^{n}$ and $S: \mathbb{R}^{n} \rightarrow \mathbb{R}^{n}$ are two directed operators and the step size $\tau$ is in the interval $\left(0, \frac{1}{\max \left\{0,\|A\|^{2}\right\}}\right)$ and proved some weak convergence theorems of the sequence $\left\{x_{n}\right\}$ to a solution of the problem (SCFPP).

In 2006, Marino and $\mathrm{Xu}$ [15] introduced a new iterative which combines the viscosity approximation method and is defined as follows:

$$
x_{n+1}=\left(I-\alpha_{n} A\right) T x_{n}+\alpha_{n} \gamma f\left(x_{n}\right)
$$

where $\left\{\alpha_{n}\right\}$ is a sequence in $(0,1)$ satisfying suitable conditions. They proved that $\left\{x_{n}\right\}$ converges strongly to a fixed point $x$ of $T$ which solves the variational inequality

$$
\langle(A-\gamma f) x, x-z\rangle \leq 0, \quad z \in F(T) .
$$


Equivalently, $P_{F(T)}(I-A+\gamma f) x=x$.

Inspired by the work mentioned above, we propose a new self-adaptive algorithm for solving the (SCFPP) with two demicontractive mappings in Hilbert spaces. We prove a strong convergence theorem for our proposed algorithm and present some numerical examples to illustrate our main results and their applications.

\section{Preliminaries}

Let $T: H \rightarrow H$ be a mapping. A point $x \in H$ is said to be a fixed point of $T$ provided that $T x=x$. In this paper, we denote by $F(T)$ the fixed point set of $T$. The symbols $\rightarrow$ and $\rightarrow$ denote the strong convergence and the weak convergence, respectively. The mapping $T: H \rightarrow H$ is said to be:

a) quasi-nonexpansive if

$$
\|T x-T p\| \leq\|x-p\|, \text { for all } x \in H \text { and } p \in F(T) .
$$

b) strictly pseudocontractive if there exists $k \in[0,1)$ such that

$$
\|T x-T y\|^{2} \leq\|x-y\|^{2}+k\|(x-y)-(T x-T y)\|^{2} \text {, for all } x \in H .
$$

c) pseudocontractive if

$$
\|T x-T y\|^{2} \leq\|x-y\|^{2}+\|(x-y)-(T x-T y)\|^{2} \text {, for all } x \in H .
$$

d) demicontractive (or $k$-demicontractive) if there exists $k<1$ such that

$$
\|T x-T p\|^{2} \leq\|x-p\|^{2}+k\|x-T x\|^{2} \text {, for all } x \in H \text { and } p \in F(T) .
$$

Remark 2.1. It is clear that, in a real Hilbert space $H,(2.1)$ is equivalent to

$$
\langle x-p, x-T x\rangle \geq \frac{1-k}{2}\|x-T x\|^{2}, \text { for all } x \in H \text { and } p \in F(T) .
$$

Now, we give some definitions and lemmas needed to prove our main results.

Definition 2.2. A mapping $T: H \rightarrow H$ is said to be demiclosed at 0 if, for each sequence $\left\{x_{n}\right\}$ in $H$, the conditions that the sequence $\left\{x_{n}\right\}$ converges weakly to $y$ and the sequence $\left\{T x_{n}\right\}$ converges strongly to 0 imply $T y=0$.

Lemma 2.3. Let $H$ be a real Hilbert space. Then the following results hold:

(1) $\|x+y\|^{2}=\|x\|^{2}+2\langle x, y\rangle+\|y\|^{2}$, for all $x, y \in H$. 
(2) $\|x-y\|^{2}=\|x\|^{2}-2\langle x, y\rangle+\|y\|^{2}$, for all $x, y \in H$.

(3) $\|\alpha x+(1-\alpha) y\|^{2}=\alpha\|x\|^{2}+(1-\alpha)\|y\|^{2}-\alpha(1-\alpha)\|x-y\|^{2}$, for all $x, y \in H$ and $\alpha \in \mathbb{R}$.

Lemma 2.4. [25] Let $\left\{a_{n}\right\}$ be a sequence of nonnegative real numbers satisfying the following relation:

$$
a_{n+1} \leq\left(1-\alpha_{n}\right) a_{n}+\alpha_{n} \sigma_{n}
$$

for each $n \geq 0$, where $\left\{\alpha_{n}\right\}$ is a sequence in $(0,1)$ and $\left\{\sigma_{n}\right\}$ is a sequence in $\mathbb{R}$ such that

(a) $\sum_{n=1}^{\infty} \alpha_{n}=\infty$;

(b) $\lim _{n \rightarrow \infty} \sigma_{n} \leq 0$ or $\sum_{n=0}^{\infty}\left|\sigma_{n} \alpha_{n}\right|<\infty$.

Then $\lim _{n \rightarrow \infty} a_{n}=0$.

Lemma 2.5. [16] Let $q>1$. Then the following inequality holds:

$$
a b \leq \frac{1}{q} a^{q}+\frac{q-1}{q} b^{\frac{q}{q-1}}
$$

for arbitrary positive real number $a$ and $b$.

\section{The Main Results}

In this section, we first construct an iterative algorithm for solving the SCFPP under the following hypotheses.

(A1) $H_{1}$ and $H_{2}$ are two real Hilbert spaces;

(A2) $A: H_{1} \rightarrow H_{2}$ is a bounded linear operator with its adjoint operator $A^{*}$.

(A3) $D: H_{1} \rightarrow H_{1}$ is a strongly positive bounded linear operator with coefficient $r>0$.

(A4) $f: H_{1} \rightarrow H_{1}$ is a $k$-contraction;

(A5) $S: H_{1} \rightarrow H_{1}$ and $T: H_{2} \rightarrow H_{2}$ are two demicontractive operators with coefficients $\beta \in[0,1)$ and $\mu \in[0,1)$, respectively;

(A6) $S: H_{1} \rightarrow H_{1}$ and $T: H_{2} \rightarrow H_{2}$ are Lipschitz continuous with Lipschitz constant $L>1$. 
We use $\Omega$ to denote the solution set of problem SCFPP, that is,

$$
\Omega:=\left\{u^{*}: u^{*} \in F(S) \text { and } A u^{*} \in F(T)\right\} .
$$

Algorithm 3.1. Choose an arbitrary initial guess $x_{0}$. Assume $x_{n}$ has been constructed. If

$$
\left\|x_{n}-S x_{n}+A^{*}(I-T) A x_{n}\right\|=0,
$$

then stop; otherwise, continue and construct $x_{n+1}$ via the formula:

$$
\left\{\begin{array}{l}
y_{n}=x_{n}-\rho_{n}\left[x_{n}-S x_{n}+A^{*}(I-T) A x_{n}\right], \\
x_{n+1}=\alpha_{n} \gamma f\left(x_{n}\right)+\left(I-\alpha_{n} D\right) y_{n},
\end{array}\right.
$$

where $\gamma \in(0, \min \{1-\beta, 1-\mu\})$ is a positive constant and $\rho_{n} \subset(0, \infty)$ is chosen self-adaptively as

$$
\rho_{n}=\sigma_{n} \frac{\left\|x_{n}-S x_{n}\right\|^{2}+\left\|A x_{n}-T A x_{n}\right\|^{2}}{\left\|x_{n}-S x_{n}+A^{*}(I-T) A x_{n}\right\|^{2}} .
$$

We need two lemmas to complete the convergence analysis of our proposed algorithm. The first lemma shows that the proposed algorithm is well defined.

Lemma 3.2. If the equality

$$
\left\|x_{n}-S x_{n}+A^{*}(I-T) A x_{n}\right\|=0,
$$

holds for some $n \geq 0$, then $x_{n}$ is a solution of problem (SCFPP).

Proof. For any $z \in S$, we have

$$
\begin{aligned}
& \left\|x_{n}-S x_{n}+A^{*}(I-T) A x_{n}\right\|\left\|x_{n}-z\right\| \\
& \geq\left\langle x_{n}-S x_{n}+A^{*}(I-T) A x_{n}\right\rangle\left\langle x_{n}-z\right\rangle \\
& =\left\langle x_{n}-S x_{n}, x_{n}-z\right\rangle+\left\langle A^{*}(I-T) A x_{n}, x_{n}-z\right\rangle \\
& =\left\langle x_{n}-S x_{n}, x_{n}-z\right\rangle+\left\langle(I-T) A x_{n}, A x_{n}-A z\right\rangle \\
& \geq \frac{1-\beta}{2}\left\|x_{n}-S x_{n}\right\|^{2}+\frac{1-\mu}{2}\left\|(I-T) A x_{n}\right\|^{2} .
\end{aligned}
$$

Since $\beta, \mu \in[0,1)$, we deduce $x_{n} \in F(S)$ and $A x_{n} \in F(T)$.

Lemma 3.3. If the sequence $\left\{x_{n}\right\}$ satisfies

$$
\lim _{n \rightarrow \infty} \frac{\left(\left\|x_{n}-S x_{n}\right\|^{2}+\left\|A x_{n}-T A x_{n}\right\|^{2}\right)^{2}}{\left\|x_{n}-S x_{n}+A^{*}(I-T) A x_{n}\right\|^{2}}=0
$$

then

$$
\lim _{n \rightarrow \infty}\left\|x_{n}-S x_{n}\right\|=\lim _{n \rightarrow \infty}(I-T) A x_{n}=0 .
$$


Proof. By our hypotheses, we have

$$
\begin{aligned}
& \frac{\left(\left\|x_{n}-S x_{n}\right\|^{2}+\left\|A x_{n}-T A x_{n}\right\|^{2}\right)^{2}}{\left\|x_{n}-S x_{n}+A^{*}(I-T) A x_{n}\right\|^{2}} \\
& \geq \frac{\left(\left\|x_{n}-S x_{n}\right\|^{2}+\left\|A x_{n}-T A x_{n}\right\|^{2}\right)^{2}}{2\left(\left\|x_{n}-S x_{n}\right\|^{2}+\|A\|^{2}\left\|(I-T) A x_{n}\right\|^{2}\right)} \\
& \geq \frac{\left(\left\|x_{n}-S x_{n}\right\|^{2}+\left\|A x_{n}-T A x_{n}\right\|^{2}\right)^{2}}{2 \max \left(1,\|A\|^{2}\right)\left(\left\|x_{n}-S x_{n}\right\|^{2}+\left\|A x_{n}-T A x_{n}\right\|^{2}\right)} \\
& =\frac{\left\|x_{n}-S x_{n}\right\|^{2}+\left\|A x_{n}-T A x_{n}\right\|^{2}}{2 \max \left(1,\|A\|^{2}\right)} .
\end{aligned}
$$

Taking $n \rightarrow \infty$, we have

$$
\lim _{n \rightarrow \infty}\left\|x_{n}-S x_{n}\right\|=\lim _{n \rightarrow \infty}(I-T) A x_{n}=0 .
$$

Theorem 3.4. Assume the following conditions are satisfied

(i) $\sum_{n=1}^{\infty} \rho_{n}=\infty$ and $\sum_{n=1}^{\infty} \rho_{n}^{2}<\infty$;

(ii) $0<r<\frac{1}{\alpha_{n}}, 0<\gamma<\frac{r}{k}$.

Then the sequence $\left\{x_{n}\right\}$ generated by Algorithm 3.1 converges strongly to a solution $u^{*}$ of problem SCFPP, where $u^{*}=P_{\Omega}(I-D+\gamma f) u^{*}$.

Proof. Setting $w_{n}=x_{n}-S x_{n}+A^{*}(I-T) A x_{n}$. Analogously, we have

$$
\begin{aligned}
\left\langle w_{n}, x_{n}-u^{*}\right\rangle & =\left\langle x_{n}-S x_{n}+A^{*}(I-T) A x_{n}, x_{n}-u^{*}\right\rangle \\
& =\left\langle x_{n}-S x_{n}, x_{n}-u^{*}\right\rangle+\left\langle A^{*}(I-T) A x_{n}, x_{n}-u^{*}\right\rangle \\
& =\left\langle x_{n}-S x_{n}, x_{n}-u^{*}\right\rangle+\left\langle(I-T) A x_{n}, A x_{n}-A u^{*}\right\rangle \\
& \geq \frac{1-\beta}{2}\left\|x_{n}-S x_{n}\right\|^{2}+\frac{1-\mu}{2}\left\|(I-T) A x_{n}\right\|^{2} \\
& \geq \frac{1}{2} \min \{1-\beta, 1-\mu\}\left(\left\|x_{n}-S x_{n}\right\|^{2}+\left\|(I-T) A x_{n}\right\|^{2}\right) .
\end{aligned}
$$

From $y_{n}=x_{n}-\rho_{n}\left[x_{n}-S x_{n}+A^{*}(I-T) A x_{n}\right]$ and (3.1), we have 


$$
\begin{aligned}
\left\|y_{n}-u^{*}\right\|^{2}= & \left\|x_{n}-\rho_{n} w_{n}-u^{*}\right\|^{2} \\
\leq & \left\|x_{n}-u^{*}\right\|^{2}-2 \rho_{n}\left\langle w_{n}, x_{n}-u^{*}\right\rangle+\rho_{n}^{2}\left\|w_{n}\right\|^{2} \\
\leq & \left\|x_{n}-u^{*}\right\|^{2}+\rho_{n}^{2}\left\|x_{n}-S x_{n}+A^{*}(I-T) A x_{n}\right\|^{2} \\
& -2 \rho_{n} \frac{1}{2} \min \{1-\beta, 1-\mu\}\left(\left\|x_{n}-S x_{n}\right\|^{2}+\left\|(I-T) A x_{n}\right\|^{2}\right) \\
\leq & \left\|x_{n}-u^{*}\right\|^{2}-\frac{\left(\left\|x_{n}-S x_{n}\right\|^{2}+\left\|(I-T) A x_{n}\right\|^{2}\right)^{2}}{\left\|x_{n}-S x_{n}+A^{*}(I-T) A x_{n}\right\|^{2}} .
\end{aligned}
$$

In particular, we have $\left\|y_{n}-u^{*}\right\| \leq\left\|x_{n}-u^{*}\right\|$. In what follows, we divide the proof into four steps.

Step 1. Show that $\left\{x_{n}\right\}$ is bounded. To see this, we observe

$$
\begin{aligned}
& \left\|x_{n+1}-u^{*}\right\|=\left\|\alpha_{n} \gamma f\left(x_{n}\right)+\left(I-\alpha_{n} D\right) y_{n}-u^{*}\right\| \\
& =\left\|\alpha_{n} \gamma\left(f\left(x_{n}\right)-D u^{*}\right)+\left(I-\alpha_{n} D\right)\left(y_{n}-u^{*}\right)\right\| \\
& \leq \alpha_{n}\left(\gamma\left\|f\left(x_{n}\right)-f\left(u^{*}\right)\right\|+\left\|\gamma f\left(u^{*}\right)-D u^{*}\right\|\right)+\left(1-\alpha_{n} r\right)\left\|y_{n}-u^{*}\right\| \\
& \leq \alpha_{n} \gamma k\left\|x_{n}-u^{*}\right\|+\alpha_{n}\left\|\gamma f\left(u^{*}\right)-D u^{*}\right\|+\left(1-\alpha_{n} r\right)\left\|y_{n}-u^{*}\right\| \\
& \leq\left(1-\alpha_{n}(r-k \gamma)\right)\left\|x_{n}-u^{*}\right\|+\alpha_{n}\left\|\gamma f\left(u^{*}\right)-D u^{*}\right\| \\
& \leq\left(1-\alpha_{n}(r-k \gamma)\right)\left\|x_{n}-u^{*}\right\|+\alpha_{n}(r-k \gamma) \frac{\left\|\gamma f\left(u^{*}\right)-D u^{*}\right\|}{r-k \gamma} \\
& \leq \max \left\{\left\|x_{n}-u^{*}\right\|, \frac{\left\|\gamma f\left(u^{*}\right)-D u^{*}\right\|}{r-k \gamma}\right\} \\
& \vdots \\
& \leq \max \left\{\left\|x_{0}-u^{*}\right\|, \frac{\left\|\gamma f\left(u^{*}\right)-D u^{*}\right\|}{r-k \gamma}\right\} .
\end{aligned}
$$

Therefore $\left\{x_{n}\right\}$ is a bounded sequence. Furthermore, $\left\{y_{n}\right\}$ and $\left\{f\left(x_{n}\right)\right\}$ are also bounded sequences.

Step 2. Show that the following inequality holds:

$$
a_{n+1} \leq\left(1-\alpha_{n}\right) a_{n}+\alpha_{n} b_{n}
$$

where we define $a_{n}:=\left\|x_{n}-u^{*}\right\|^{2}$ and 


$$
\begin{aligned}
b_{n}:= & \frac{2\left(1-\alpha_{n} \gamma k\right)}{r-\gamma k}\left\langle\gamma f\left(u^{*}\right)-D u^{*}, x_{n+1}-u^{*}\right\rangle \\
& -\frac{1-\alpha_{n} r}{\alpha_{n}(r-\gamma k)} \frac{\left(\left\|x_{n}-S x_{n}\right\|^{2}+\left\|(I-T) A x_{n}\right\|^{2}\right)^{2}}{\left\|x_{n}-S x_{n}+A^{*}(I-T) A x_{n}\right\|^{2}} .
\end{aligned}
$$

Indeed, it follows that

$$
\begin{aligned}
\left\|x_{n+1}-u^{*}\right\|^{2} & =\left\|\alpha_{n} \gamma f\left(y_{n}\right)+\left(I-\alpha_{n} D\right) y_{n}-u^{*}\right\|^{2} \\
& =\left\|\alpha_{n} \gamma\left(f\left(y_{n}\right)-D u^{*}\right)+\left(I-\alpha_{n} D\right)\left(y_{n}-u^{*}\right)\right\|^{2} \\
& \leq\left\|\left(I-\alpha_{n} D\right)\left(y_{n}-u^{*}\right)\right\|^{2}+2 \alpha_{n}\left\langle\gamma f\left(y_{n}\right)-D u^{*}, x_{n+1}-u^{*}\right\rangle \\
& \leq\left\|I-\alpha_{n} D\right\|^{2}\left\|y_{n}-u^{*}\right\|^{2}+2 \alpha_{n}\left\langle\gamma f\left(y_{n}\right)-D u^{*}, x_{n+1}-u^{*}\right\rangle \\
& \leq\left(1-\alpha_{n} r\right)^{2}\left\|y_{n}-u^{*}\right\|^{2}+2 \alpha_{n}\left\langle\gamma f\left(y_{n}\right)-D u^{*}, x_{n+1}-u^{*}\right\rangle \\
& \leq\left(1-\alpha_{n} r\right)\left\|y_{n}-u^{*}\right\|^{2}+2 \alpha_{n}\left\langle\gamma f\left(y_{n}\right)-D u^{*}, x_{n+1}-u^{*}\right\rangle .
\end{aligned}
$$

From Lemma 2.5, we have

$$
\begin{aligned}
\left\langle\gamma f\left(y_{n}\right)-D u^{*}, x_{n+1}-u^{*}\right\rangle= & \left\langle\gamma f\left(y_{n}\right)-\gamma f\left(u^{*}\right), x_{n+1}-u^{*}\right\rangle \\
& +\left\langle\gamma f\left(u^{*}\right)-D u^{*}, x_{n+1}-u^{*}\right\rangle \\
\leq & \gamma k\left\|y_{n}-u^{*}\right\|\left\|x_{n+1}-u^{*}\right\| \\
& +\left\langle\gamma f\left(u^{*}\right)-D u^{*}, x_{n+1}-u^{*}\right\rangle \\
\leq & \gamma k\left(\frac{1}{2}\left\|y_{n}-u^{*}\right\|^{2}+\frac{1}{2}\left\|x_{n+1}-u^{*}\right\|^{2}\right) \\
& +\left\langle\gamma f\left(u^{*}\right)-D u^{*}, x_{n+1}-u^{*}\right\rangle .
\end{aligned}
$$

Substitute (3.7) into (3.6), we have

$$
\begin{aligned}
\left\|x_{n+1}-u^{*}\right\|^{2} \leq & \left(1-\alpha_{n} r\right)\left\|y_{n}-u^{*}\right\|^{2}+2 \alpha_{n}\left(\gamma k\left(\frac{1}{2}\left\|y_{n}-u^{*}\right\|^{2}+\frac{1}{2}\left\|x_{n+1}-u^{*}\right\|^{2}\right)\right. \\
& \left.+\left\langle\gamma f\left(u^{*}\right)-D u^{*}, x_{n+1}-u^{*}\right\rangle\right) \\
\leq & \left(1-\alpha_{n} r\right)\left\|y_{n}-u^{*}\right\|^{2}+\alpha_{n} \gamma k\left\|y_{n}-u^{*}\right\|^{2}+\alpha_{n} \gamma k\left\|x_{n+1}-u^{*}\right\|^{2} \\
& +2 \alpha_{n}\left\langle\gamma f\left(u^{*}\right)-D u^{*}, x_{n+1}-u^{*}\right\rangle \\
\leq & \left(1-\frac{\alpha_{n}(r-\gamma k)}{1-\alpha_{n} \gamma k}\right)\left\|y_{n}-u^{*}\right\|^{2} \\
& +\frac{\alpha_{n}(r-\gamma k)}{1-\alpha_{n} \gamma k} \frac{2\left(1-\alpha_{n} \gamma k\right)}{r-\gamma k}\left\langle\gamma f\left(u^{*}\right)-D u^{*}, x_{n+1}-u^{*}\right\rangle .
\end{aligned}
$$


By inequality (3.2), this yields

$$
\begin{aligned}
\left\|x_{n+1}-u^{*}\right\|^{2} \leq & \left(1-\frac{\alpha_{n}(r-\gamma k)}{1-\alpha_{n} \gamma k}\right)\left\|x_{n}-u^{*}\right\|^{2} \\
& +\frac{\alpha_{n}(r-\gamma k)}{1-\alpha_{n} \gamma k} \frac{2\left(1-\alpha_{n} \gamma k\right)}{r-\gamma k}\left\langle\gamma f\left(u^{*}\right)-D u^{*}, x_{n+1}-u^{*}\right\rangle \\
\leq & \left(1-\frac{\alpha_{n}(r-\gamma k)}{1-\alpha_{n} \gamma k}\right)\left\|x_{n}-u^{*}\right\|^{2} \\
& +\frac{\alpha_{n}(r-\gamma k)}{1-\alpha_{n} \gamma k}\left[\frac{2\left(1-\alpha_{n} \gamma k\right)}{r-\gamma k}\left\langle\gamma f\left(u^{*}\right)-D u^{*}, x_{n+1}-u^{*}\right\rangle\right. \\
& \left.-\frac{1-\alpha_{n} r}{\alpha_{n}(r-\gamma k)} \frac{\left(\left\|x_{n}-S x_{n}\right\|^{2}+\left\|(I-T) A x_{n}\right\|^{2}\right)^{2}}{\left\|x_{n}-S x_{n}+A^{*}(I-T) A x_{n}\right\|^{2}}\right] .
\end{aligned}
$$

Hence, the desired inequality at once follows. Step 3. Show that $-\delta \leq$ $\overline{\lim }_{n \rightarrow \infty} b_{n}<+\infty$ for some $\delta>0$, which indicate that $\overline{\lim }_{n \rightarrow \infty} b_{n}$ is finite. Since $\left\{x_{n}\right\}$ is bounded, we have

$$
\begin{aligned}
b_{n} & \leq \frac{2\left(1-\alpha_{n} \gamma k\right)}{r-\gamma k}\left\langle\gamma f\left(u^{*}\right)-D u^{*}, x_{n+1}-u^{*}\right\rangle \\
& \leq \frac{2\left(1-\alpha_{n} \gamma k\right)}{r-\gamma k}\left\|\gamma f\left(u^{*}\right)-D u^{*}\right\|\left\|x_{n+1}-u^{*}\right\| \\
& <+\infty
\end{aligned}
$$

so that this implies $-\delta \leq \overline{\lim }_{n \rightarrow \infty} b_{n}<+\infty$. We next prove $\overline{\lim }_{n \rightarrow \infty} b_{n} \geq-\delta$. To this aim, we processed by contradiction. Assume that $\varlimsup_{n \rightarrow \infty} b_{n}<-\delta$, which implies that there exists $n_{0} \in \mathbb{N}$ such that $b_{n} \leq-\delta$ for all $n \geq n_{0}$, it follows from that

$$
\begin{aligned}
a_{n+1} & \leq\left(1-\alpha_{n}\right) a_{n}+\alpha_{n} b_{n} \\
& \leq\left(1-\alpha_{n}\right) a_{n}-\alpha_{n} \delta \\
& =a_{n}-\alpha_{n}\left(a_{n}+\delta\right) \\
& \leq a_{n}-\frac{\alpha_{n}(1-\gamma k) \delta}{1-\alpha_{n} \gamma k} .
\end{aligned}
$$

for all $n \geq n_{0}$. By induction, we have

$$
a_{n+1} \leq a_{n_{0}}-\left(\frac{(1-\gamma k) \delta \sum_{i=n_{0}}^{n} \alpha_{i}}{1-\gamma k \sum_{i=n_{0}}^{n} \alpha_{i}}\right)
$$

Hence, taking $\varlimsup$ as $n \rightarrow \infty$ in the last inequality, we have

$$
\varlimsup_{n \rightarrow \infty} a_{n} \leq \varlimsup_{n \rightarrow \infty}\left[a_{n_{0}}-\left(\frac{(1-\gamma k) \delta \sum_{i=n_{0}}^{n} \alpha_{i}}{1-\gamma k \sum_{i=n_{0}}^{n} \alpha_{i}}\right)\right]=-\infty
$$


which clearly contradicts the fact that $\left\{a_{n}\right\}$ is a nonnegative real sequence. Thus, $\varlimsup_{n \rightarrow \infty} b_{n} \geq-\delta$ and it is finite.

Step 4. Show that $\left\{x_{n}\right\}$ converges to $z$. Since $\varlimsup_{n \rightarrow \infty} b_{n}$ is finite, we can take a subsequence $\left\{n_{k}\right\}$ such that

$$
\begin{aligned}
\varlimsup_{n \rightarrow \infty} b_{n}= & \lim _{k \rightarrow \infty} b_{n_{k}} \\
= & \lim _{k \rightarrow \infty}\left[\frac{2\left(1-\alpha_{n} \gamma k\right)}{r-\gamma k}\left\langle\gamma f\left(u^{*}\right)-D u^{*}, x_{n_{k}+1}-u^{*}\right\rangle\right. \\
& \left.-\frac{1-\alpha_{n} r}{\alpha_{n}(r-\gamma k)} \frac{\left(\left\|x_{n_{k}}-S x_{n_{k}}\right\|^{2}+\left\|(I-T) A x_{n_{k}}\right\|^{2}\right)^{2}}{\left\|x_{n_{k}}-S x_{n_{k}}+A^{*}(I-T) A x_{n_{k}}\right\|^{2}}\right] .
\end{aligned}
$$

Since $\left\langle\gamma f\left(u^{*}\right)-D u^{*}, x_{n_{k}+1}-u^{*}\right\rangle$ is a bounded real sequence, without loss of generality, we may assume there exists the limit:

$$
\lim _{k \rightarrow \infty}\left\langle\gamma f\left(u^{*}\right)-D u^{*}, x_{n_{k}+1}-u^{*}\right\rangle .
$$

Consequently, from (3.14), the following limit also exists:

$$
\lim _{k \rightarrow \infty} \frac{1-\alpha_{n} r}{\alpha_{n}(r-\gamma k)} \frac{\left(\left\|x_{n_{k}}-S x_{n_{k}}\right\|^{2}+\left\|(I-T) A x_{n_{k}}\right\|^{2}\right)^{2}}{\left\|x_{n_{k}}-S x_{n_{k}}+A^{*}(I-T) A x_{n_{k}}\right\|^{2}}
$$

which implies that the sequence

$$
\frac{1}{\alpha_{n}(r-\gamma k)} \frac{\left(\left\|x_{n_{k}}-S x_{n_{k}}\right\|^{2}+\left\|(I-T) A x_{n_{k}}\right\|^{2}\right)^{2}}{\left\|x_{n_{k}}-S x_{n_{k}}+A^{*}(I-T) A x_{n_{k}}\right\|^{2}}
$$

is bounded. So, by condition $\alpha_{n} \rightarrow 0$, we have

$$
\lim _{k \rightarrow \infty} \frac{\left(\left\|x_{n_{k}}-S x_{n_{k}}\right\|^{2}+\left\|(I-T) A x_{n_{k}}\right\|^{2}\right)^{2}}{\left\|x_{n_{k}}-S x_{n_{k}}+A^{*}(I-T) A x_{n_{k}}\right\|^{2}}=0 .
$$

By Lemma 3.3, we have

$$
\lim _{n_{k} \rightarrow \infty}\left\|x_{n_{k}}-S x_{n_{k}}\right\|=\lim _{n_{k} \rightarrow \infty}\left\|A x_{n_{k}}-T A x_{n_{k}}\right\|=0 .
$$

By the definition of $x_{n_{k}+1}$, we deduce that

$$
\begin{aligned}
\lim _{k \rightarrow \infty}\left\|x_{n_{k}}-y_{n_{k}}\right\| & =\lim _{k \rightarrow \infty} \rho_{n_{k}}\left\|x_{n_{k}}-S x_{n_{k}}+A^{*}(I-T) A x_{n_{k}}\right\| \\
& =\lim _{k \rightarrow \infty} \frac{\left(\left\|x_{n_{k}}-S x_{n_{k}}\right\|^{2}+\left\|(I-T) A x_{n_{k}}\right\|^{2}\right)^{2}}{\left\|x_{n_{k}}-S x_{n_{k}}+A^{*}(I-T) A x_{n_{k}}\right\|^{2}}=0
\end{aligned}
$$


which further implies

$$
\begin{aligned}
\left\|x_{n_{k}+1}-x_{n_{k}}\right\| & =\left\|\alpha_{n_{k}} \gamma f\left(x_{n_{k}}\right)+\left(I-\alpha_{n_{k}} B\right) y_{n_{k}}-x_{n_{k}}\right\| \\
& \leq \alpha_{n_{k}}\left\|\gamma f\left(x_{n_{k}}\right)-D x_{n_{k}}\right\|+\left(1-\alpha_{n_{k}} r\right)\left\|y_{n_{k}}-x_{n_{k}}\right\| \\
& \rightarrow 0 .
\end{aligned}
$$

Since we have shown that the sequence $\left\{x_{n}\right\}$ is bounded. This implies that any weak cluster point of $\left\{x_{n_{k}+1}\right\}$ also belongs to $\Omega$. Without loss of generality, we assume that $\left\{x_{n_{k}+1}\right\}$ converges weakly to $x \in \Omega$. Now by (3.14), we infer that

$$
\begin{aligned}
\varlimsup_{n \rightarrow \infty} b_{n} & =\lim _{k \rightarrow \infty} \frac{2\left(1-\alpha_{n} \gamma k\right)}{r-\gamma k}\left\langle\gamma f\left(u^{*}\right)-D u^{*}, x_{n_{k}+1}-u^{*}\right\rangle \\
& =\frac{2\left(1-\alpha_{n} \gamma k\right)}{r-\gamma k}\left\langle\gamma f\left(u^{*}\right)-D u^{*}, x-u^{*}\right\rangle \leq 0
\end{aligned}
$$

due to the fact that $u^{*}=P_{\Omega}(I-D+\gamma f) u^{*}$ and (2.3). Finally, applying Lemma 2.4 to (3.4), we arrive at $\left\|x_{n}-u^{*}\right\| \rightarrow 0$, which ends the proof.

In the cases $f\left(x_{n}\right)=u$, we have the algorithm as follows

Algorithm 3.5. Choose an arbitrary initial guess $u, x_{0}$. Assume $x_{n}$ has been constructed. If

$$
\left\|x_{n}-S x_{n}+A^{*}(I-T) A x_{n}\right\|=0,
$$

then stop; otherwise, continue and construct $x_{n+1}$ via the formula:

$$
\left\{\begin{array}{l}
y_{n}=x_{n}-\rho_{n}\left[x_{n}-S x_{n}+A^{*}(I-T) A x_{n}\right] \\
x_{n+1}=\alpha_{n} \gamma u+\left(I-\alpha_{n} D\right) y_{n}
\end{array}\right.
$$

where $\gamma \in(0, \min \{1-\beta, 1-\mu\})$ is a positive constant and $\rho_{n} \subset(0, \infty)$ is chosen self-adaptively as

$$
\rho_{n}=\sigma_{n} \frac{\left\|x_{n}-S x_{n}\right\|^{2}+\left\|A x_{n}-T A x_{n}\right\|^{2}}{\left\|x_{n}-S x_{n}+A^{*}(I-T) A x_{n}\right\|^{2}} .
$$

Corollary 3.6. Assume the following conditions are satisfied

(i) $\sum_{n=1}^{\infty} \rho_{n}=\infty$ and $\sum_{n=1}^{\infty} \rho_{n}^{2}<\infty$;

(ii) $0<r<\frac{1}{\alpha_{n}}, 0<\gamma<r$.

Then the sequence $\left\{x_{n}\right\}$ generated by Algorithm 3.5 converges strongly to a solution $u^{*}$ of problem SCFPP, where $u^{*}=P_{\Omega}\left(u^{*}-D u^{*}+u\right)$. 


\section{Numerical experiments}

In this section, we construct a numerical example to illustrate the algorithm (3.1) and convergence analysis of the sequences of our main result. All codes were written in Matlab 2018a and run on Dell i-5 Core laptop.

Example 4.1. Let $H_{1}=H_{2}=\left(\mathbb{R}^{2},\|\cdot\|_{2}\right)$. Define the mappings $S: \mathbb{R}^{2} \rightarrow \mathbb{R}^{2}$ and $T: \mathbb{R}^{2} \rightarrow \mathbb{R}^{2}$ by

$$
S\left(x_{1}, y_{1}\right)=\left(-3 x_{1}, y_{1}\right) \quad \text { and } \quad T\left(x_{1}, y_{1}\right)=-5\left(x_{1}, y_{1}\right), \quad \forall x_{1}, y_{1} \in \mathbb{R} .
$$

First, we show that $S$ is a $\frac{1}{2}$-demicontractive mapping. if $x=\left(x_{1}, y_{1}\right) \in \mathbb{R}^{2}$ and $p_{1}=(0, a) \in F(S)$, then

$$
\begin{aligned}
\left\|S x-p_{1}\right\|_{2}^{2} & =\left\|\left(-3 x_{1}, y_{1}\right)-(0, a)\right\|_{2}^{2} \\
& =(-3)^{2}\left|x_{1}\right|^{2}+\left|y_{1}-a\right|^{2} \\
& =9\left|x_{1}\right|^{2}+\left|y_{1}-p_{1}\right|^{2} \\
& =\left|x_{1}\right|^{2}+\left|y_{1}-a\right|^{2}+8\left|x_{1}\right|^{2} \\
& =\left\|x-p_{1}\right\|_{2}^{2}+\frac{8}{16}\left(16\left|x_{1}\right|^{2}\right) \\
& =\left\|x-p_{1}\right\|_{2}^{2}+\frac{1}{2}\|x-S x\|_{2}^{2} .
\end{aligned}
$$

Thus, $S$ is a $\frac{1}{2}$-demicontractive mapping.

Second, we show that $T$ is a $\frac{2}{3}$-demicontractive mapping. if $x=\left(x_{1}, y_{1}\right) \in \mathbb{R}^{2}$ and $p_{2}=(0,0) \in F(T)$, then

$$
\begin{aligned}
\left\|T x-p_{2}\right\|_{2}^{2} & =\left\|-5\left(x_{1}, y_{1}\right)-(0,0)\right\|_{2}^{2} \\
& =25\left|x_{1}\right|^{2}+25\left|y_{1}\right|^{2} \\
& =\left|x_{1}\right|^{2}+\left|y_{1}\right|^{2}+24\left(\left|x_{1}\right|^{2}+\left|y_{1}\right|^{2}\right) \\
& =\left\|x-p_{2}\right\|_{2}^{2}+\frac{24}{36}\left(36\left(\left|x_{1}\right|^{2}+\left|y_{1}\right|^{2}\right)\right) \\
& =\left\|x-p_{2}\right\|_{2}^{2}+\frac{2}{3}\|x-T x\|_{2}^{2} .
\end{aligned}
$$

Thus, $T$ is a $\frac{2}{3}$-demicontractive mapping. Next, we define the mappings $f: \mathbb{R}^{2} \rightarrow \mathbb{R}^{2}, A: \mathbb{R}^{2} \rightarrow \mathbb{R}^{2}$ and $D: \mathbb{R}^{2} \rightarrow \mathbb{R}^{2}$ by

$f\left(x_{1}, y_{1}\right)=\left(\frac{x_{1}}{8}, \frac{y_{1}}{8}\right), A\left(x_{1}, y_{1}\right)=8\left(x_{1}, y_{1}\right), D\left(x_{1}, y_{1}\right)=\left(\frac{x_{1}}{2}, \frac{y_{1}}{2}\right), \forall x_{1}, y_{1} \in \mathbb{R}$.

Then $f$ is a $\frac{1}{8}$-contraction, $A$ is a bounded linear operator on $\mathbb{R}$ with adjoint operator $A^{*}$ and $D$ is a strongly positive bounded linear operator with coefficient $\xi=\frac{1}{2}$. In Algorithm (3.1), we set $\sigma_{n}=0.15, \gamma=0.1$ and $\alpha_{n}=\frac{1}{10 n+100}$. 
We compare with Moudafi in [27], by set $\gamma=0.0018$ and $\alpha_{n}=\frac{1}{10 n+100}$. Then, we have the results in Table 4.1 and Figure 4.1.

Table 4.1: Result of Example 4.1.

\begin{tabular}{cccc}
\hline & & & \\
$\left(x_{1}, y_{1}\right)$ & & Algorithm 3.1 & Moudafi \\
\hline \multirow{4}{*}{$(-9,9)$} & No. of Iter. & 6 & 15 \\
& Approximation & $(-0.000000,0.000000)$ & $(-0.000000,0.000000)$ \\
& $\left\|x_{n+1}-x_{n}\right\|_{2}$ & 0.000000 & 0.000000 \\
& Time & 0.036015 & 0.025440 \\
\hline \multirow{4}{*}{$(20,10)$} & No. of Iter. & 6 & 15 \\
& Approximatio & $(0.000000,0.000000)$ & $(0.000000,0.000000)$ \\
& $\left\|x_{n+1}-x_{n}\right\|_{2}$ & 0.000000 & 0.000001 \\
& Time & 0.027071 & 0.015959 \\
\hline \multirow{4}{*}{$(-6,-2)$} & No. of Iter. & 6 & 14 \\
& Approximatio & $(0.000000,-0.000000)$ & $(-0.000000,-0.000000)$ \\
& $\left\|x_{n+1}-x_{n}\right\|_{2}$ & 0.000000 & 0.000001 \\
& Time & 0.036619 & 0.015571 \\
\hline
\end{tabular}

\subsection{Compressed sensing}

Compressed sensing is a very active domain of research and applications, based on the fact that an $K$-sample signal $x$ with $M \leq N<K$. The sampling matrix $A \in \mathbb{R}^{M \times N}(M<N)$ is stimulated by standard Gaussian distribution and vector $z=A x_{0}+b$, where $b$ is additive noise. The most common form of disorder technique is $l_{1}$ regularization as:

$$
\min _{x \in \mathbb{R}^{N}}\left\{\frac{1}{2}\|A x-z\|^{2}+\alpha\|x\|_{1}\right\}
$$

where is $\alpha$ a positive parameter and $\|\cdot\|_{1}$ denotes the sum of the absolute values of the components. By means of convex analysis, one is able to show that a solution to the constrained least squares problem:

$$
\min _{x \in \mathbb{R}^{N}}\left\{\frac{1}{2}\|A x-z\|^{2}\right\} \quad \text { subjet to } \quad\|x\|_{1} \leq t,
$$

for any nonnegative real number $t$, is a minimizer of (4.1) (see [9]). Clearly problem (4.2) is a particular case of problem (SFP) where $C=\left\{x \in \mathbb{R}^{N}\right.$ : 


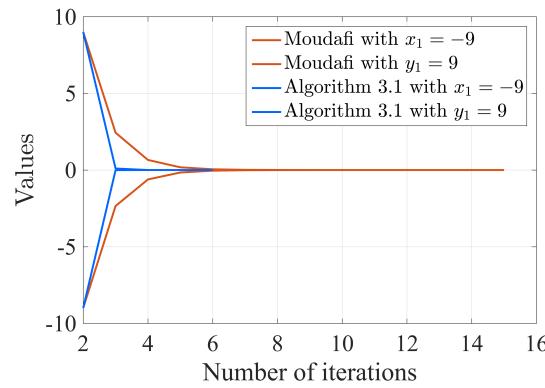

(a) $\left(x_{1}, y_{1}\right)=(-9,9)$

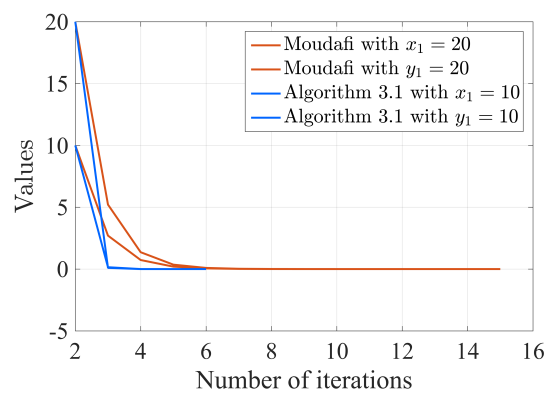

(c) $\left(x_{1}, y_{1}\right)=(20,10)$

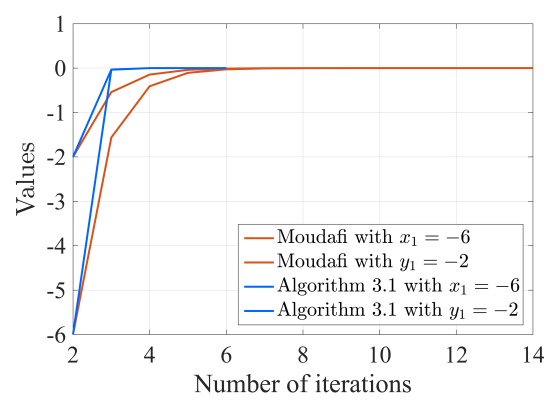

(e) $\left(x_{1}, y_{1}\right)=(-6,-2)$

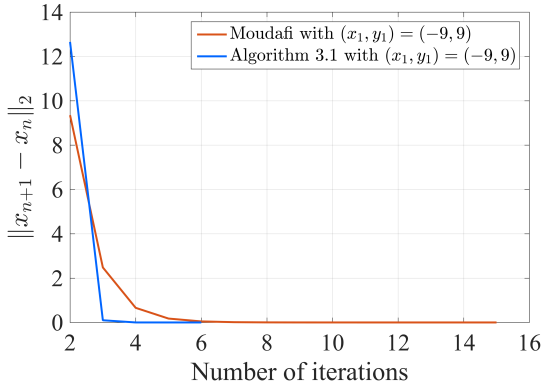

(b) $\left(x_{1}, y_{1}\right)=(-9,9)$

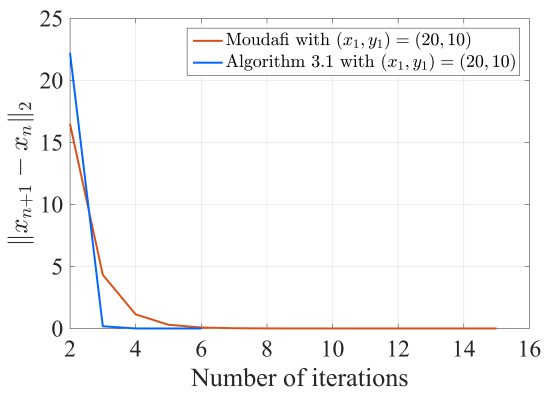

(d) $\left(x_{1}, y_{1}\right)=(20,10)$

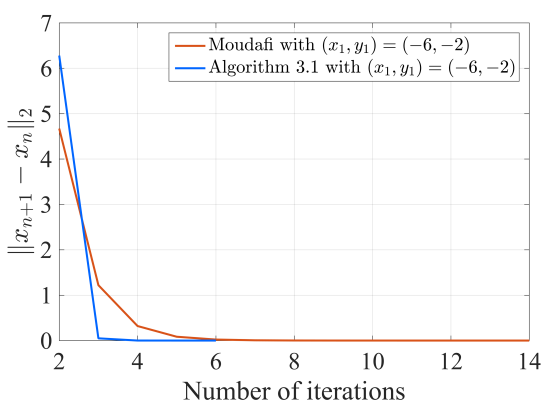

(f) $\left(x_{1}, y_{1}\right)=(-6,-2)$

Figure 4.1: Result of Example 4.1. 
$\left.\|x\|_{1} \leq t\right\}$ and $Q=\{z\}$. and thus can be solved by the proposed algorithm. In this case, $P_{C}$ is the projection onto the closed $l_{1}$-ball in $\mathbb{R}^{n}$ (see [7]).

Algorithm 4.2. Choose an arbitrary initial guess $x_{0}$. Assume $x_{n}$ has been constructed. If

$$
\left\|x_{n}-P_{C} x_{n}+A^{*}\left(A x_{n}-z\right)\right\|=0,
$$

then stop; otherwise, continue and construct $x_{n+1}$ via the formula:

$$
\left\{\begin{array}{l}
y_{n}=x_{n}-\rho_{n}\left[x_{n}-P_{C} x_{n}+A^{*}\left(A x_{n}-z\right)\right], \\
x_{n+1}=\alpha_{n} \gamma f\left(x_{n}\right)+\left(I-\alpha_{n} D\right) y_{n},
\end{array}\right.
$$

where $\gamma \in(0,1)$ is a positive constant, $\sigma_{n} \in(0,1)$ and $\rho_{n} \in(0,1)$ is chosen self-adaptively as

$$
\rho_{n}=\sigma_{n} \frac{\left\|x_{n}-P_{C} x_{n}\right\|^{2}+\left\|A x_{n}-z\right\|^{2}}{\left\|x_{n}-P_{C} x_{n}+A^{*}\left(A x_{n}-z\right)\right\|^{2}} .
$$

Theorem 4.3. Let $\left\{x_{n}\right\}$ be the sequence generated by Algorithm 4.2. If the sequence $\left\{\rho_{n}\right\}$ satisfies $\sum_{n=1}^{\infty} \rho_{n}=\infty$ and $\sum_{n=1}^{\infty} \rho_{n}^{2}<\infty$, then $\left\{x_{n}\right\}$ converges weakly to a solution $u^{*}$ of split feasibility problem .

Proof. Take $S=P_{C}$ and $T=P_{Q}$ in Theorem 3.4.

In our experiment, we set the hits of a signal $x \in \mathbb{R}^{N}$ is $N=2^{12}$. There exist $K=50$ spikes with amplitude \pm 1 distributed in the whole domain randomly. Then we set the observation dimension $M=2^{10}$ with white Gaussian noise of variance $\epsilon^{2}=10^{-4}$. The process is started with initial signal $x_{0}=A^{*} z$ and finishes with 400 iterations. The restoration accuracy is measured by means of the mean squared error: MSE $=\frac{\left\|x^{*}-x\right\|^{2}}{N}$, where $x^{*}$ is an estimated signal of $x$. All codes were written in Matlab 2018a and run on Dell i-5 Core laptop. We compare the performances of Algorithm 4.2 by $f(x)=\frac{1}{8} x, D(x)=\frac{1}{2} x, \sigma_{n}=$ $0.15, \gamma=0.1$ and $\alpha_{n}=\frac{1}{10 n+100}$. with Byrne's algorithm [2] by $\gamma=0.1$ are reported in Figure 4.2.

\subsection{Image restoration}

We apply the algorithm 4.2 in the paper to image restoration. The observation model can also be described as (4.1), we wish to estimate an original image $x$ from an observation $z$, while matrix $A$ represents the blur operator ('motion',15,60), and $b$ is random noise. The signal to noise ratio (SNR) is used to measure the quality of the restored images. They are defined as follows:

$$
\mathrm{SNR}=20 \log \frac{\|x\|}{\left\|x-x_{n}\right\|}
$$



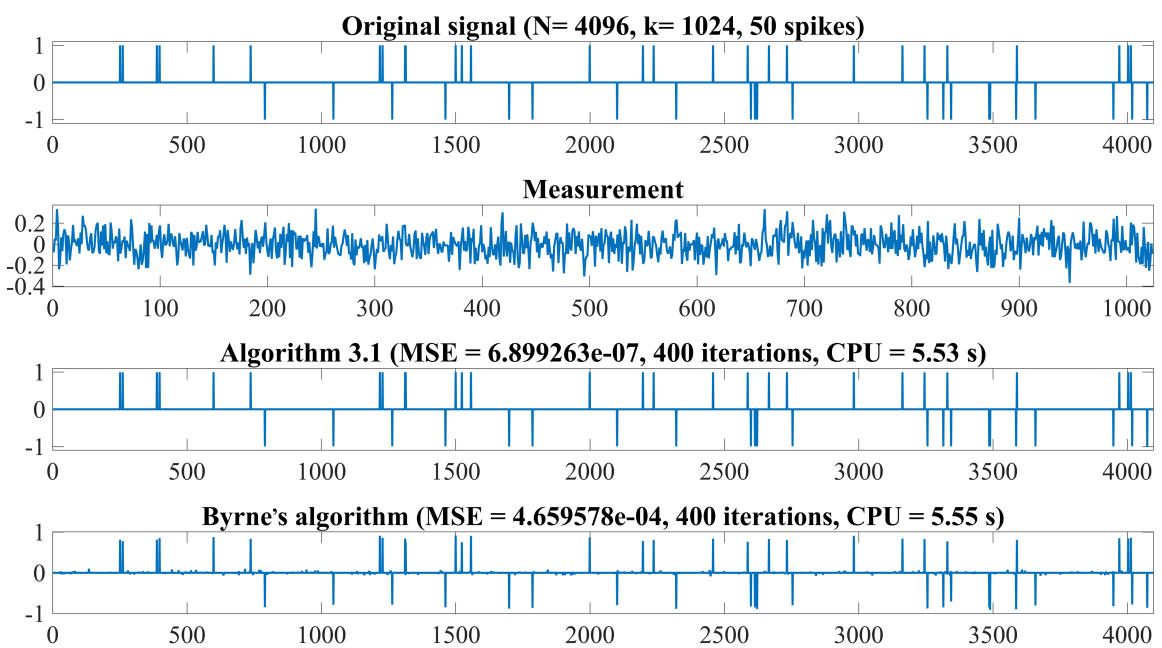

Figure 4.2: From top to bottom: original signal, reconstruction signals respectively by Algorithm 4.2 and Byrne's algorithm

where $x, z$ and $x_{n}$ are the original image, the observed image and estimated image at iteration $n$, respectively. The process is started with initial signal $x_{0}=A x+b$ and finishes with 100 iterations. All codes were written in Matlab 2018a and run on Dell i-5 Core laptop.

We compare the performances of Algorithm 4.2 by $f(x)=\frac{1}{8} x, D(x)=\frac{1}{2} x, \sigma_{n}=$ $0.2, \gamma=0.9$ and $\alpha_{n}=\frac{1}{10 n+100}$ with Byrne's algorithm [2] by $\gamma=0.9$ are reported in Figure 4.3 and Figure 4.4 .

\section{Conclusions}

In this article, we proposed a new iterative scheme for finding common solutions of demicontractive operators. Under some suitable conditions imposed on parameters, we proved some strong convergence theorems of the proposed algorithm and, finally, we presented some numerical results to show that our algorithm performs better than some existing methods.

\section{Acknowledgments}

The first author thanks the support of Petchra Pra Jom Klao Doctoral Scholarship for PhD Program at King Mongkut's University of Technology Thonburi (KMUTT). This work was completed while the first and last authors visited 


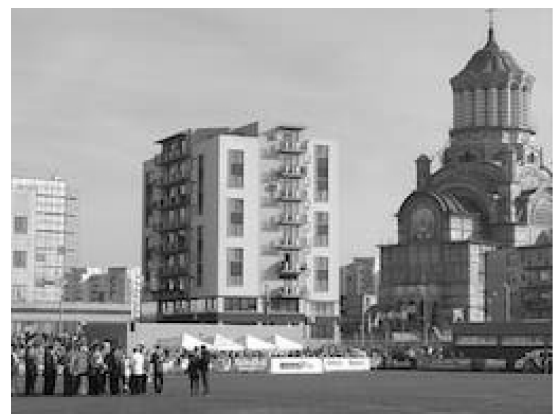

(a) original image

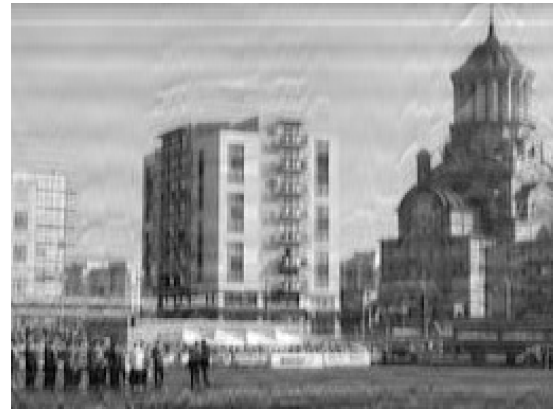

(c) Algorithm $4.2(\mathrm{SNR}=50.30,100$ iter, $(\mathrm{d})$ Byrne's algorithm $(\mathrm{SNR}=46.35,100$ $\mathrm{CPU}=8.63 \mathrm{~s})$

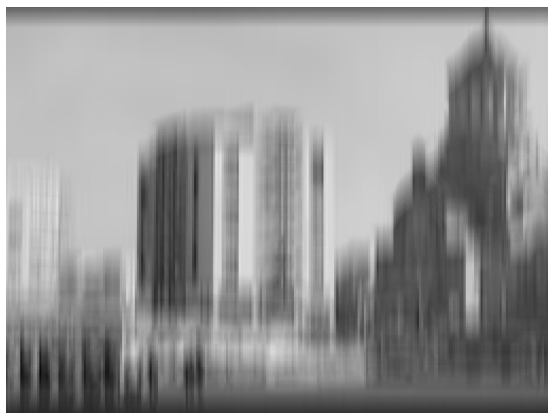

(b) blur and noisy image

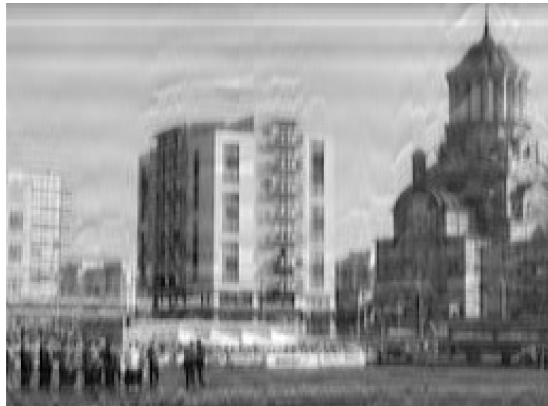
iter, $\mathrm{CPU}=5.01 \mathrm{~s})$

Figure 4.3: Result of restoration image size $192 \times 256$ : (a) original image, (b) blur and noisy image, (c) restoration by Algorithm 4.2 and (d) restoration by Byrne' s algorithm 


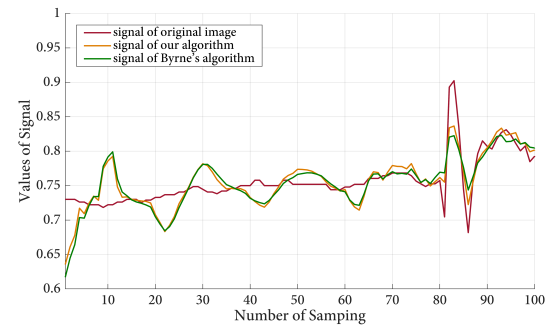

(a) signal image at $(x, y)=(:, 10)$

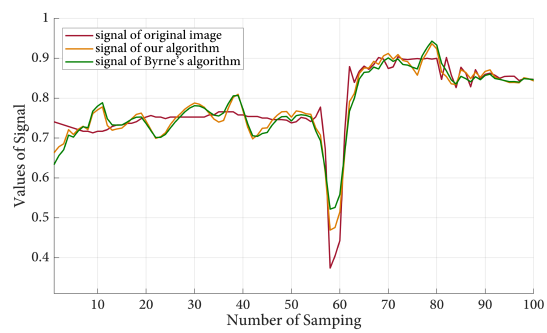

(c) signal image at $(x, y)=(:, 100)$

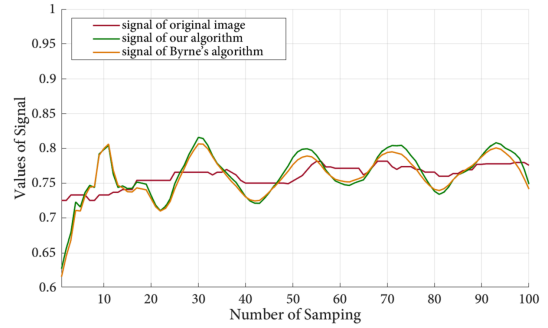

(b) signal image at $(x, y)=(:, 50)$

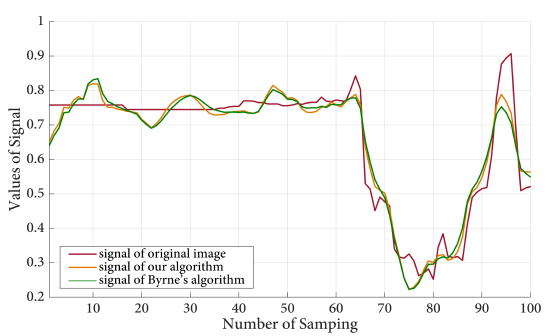

(d) signal image at $(x, y)=(:, 150)$

Figure 4.4: Signal of restoration: (a) signal of position $(x, y)=(:, 10)$, (b) signal of position $(x, y)=(:, 50)$, (c) signal of position $(x, y)=(:, 100)$ and $(\mathrm{d})$ signal of position $(x, y)=(:, 150)$ 
Prof. Vasile Berinde at North University Center of Baia Mare (Technical University of Cluj-Napoca). They thank very much Prof. Vasile Berinde for his hospitality and support. Finally, Poom Kumam was supported by the Thailand Research Fund (TRF) and the King Mongkut's University of Technology Thonburi (KMUTT) under the TRF Research Scholar Award (Grant No.RSA6080047).

\section{References}

[1] Ansari, Q. H., Rehan, A., Iterative methods for generalized split feasibility problems in Banach spaces. Carpathian J. Math. 33 (2017), no. 1, $9-26$.

[2] Byrne, C.: Iterative oblique projection onto convex sets and the split feasibility problem, Inverse Problems 18, 441-453 (2002).

[3] Censor, Y., Segal, A.: The split common fixed point problem for directed operators. J. Convex Anal.16(2), 587-600 (2009).

[4] Chidume, C. E., Nnakwe, M. O., A new Halpern-type algorithm for a generalized mixed equilibrium problem and a countable family of generalized nonexpansive-type maps. Carpathian J. Math. 34 (2018), no. 2, 191-198.

[5] Chuasuk, P., Farajzadeh, A., Kaewcharoen, A., Agarwal, R. P., An iterative process for a hybrid pair of a Bregman strongly nonexpansive single-valued mapping and a finite family of Bregman relative nonexpansive multi-valued mappings in Banach spaces. Carpathian J. Math. 33 (2017), no. 3, 287-300.

[6] Dogan, K., Karakaya, V., Approximation of fixed point of accretive operators based on a Halpern-type iterative method. Creat. Math. Inform. 26 (2017), no. 3, 263-274.

[7] Duchi, J.C., Shalevshwartz, S., Singer, Y. et al. Efficient projections onto the $l_{1}$-ball for learning in high dimensions, International conference on machine learning, 2008: 272-279.

[8] Farajzadeh, A., Chuasuk, P., Kaewcharoen, A., Mursaleen, M., An iterative process for a hybrid pair of generalized I-asymptotically nonexpansive single-valued mappings and generalized nonexpansive multi-valued mappings in Banach spaces. Carpathian J. Math. 34 (2018), no. 1, 31-45. 
[9] Figueiredo, M. A., Nowak, R. D., Wright, S. J.: Gradient projection for sparse reconstruction: Application to Compressed Sensing and other Inverse Problems, IEEE J. Sel. Top. Signal Process. 1 (2007), 586-598.

[10] He, S., Yang, C.: Solving the variational inequality problem defined on intersection of finite level sets. Abstr. Appl. Anal. 2013, Art ID 942315 (2013).

[11] Hung, N. V., Hoang, D. H., Tam, V. M., Painlevé-Kuratowski convergences of the approximate solution sets for vector quasiequilibrium problems. Carpathian J. Math. 34 (2018), no. 1, 115-122.

[12] Kazmi, K. R., Rizvi, S. H., Ali, Rehan, A hybrid iterative method without extrapolating step for solving mixed equilibrium problem. Creat. Math. Inform. 24 (2015), no. 2, 163-170.

[13] Khan, A. R., Fukhar-ud-din, H., Yasmin, N., Approximating common fixed point of asymptotically nonexpansive mappings without convergence condition. Carpathian J. Math. 33 (2017), no. 3, 327-334.

[14] Meche, T. H., Sangago, M. G., Zegeye, H., Iterative methods for a fixed point of hemicontractive-type mapping and a solution of a variational inequality problem. Creat. Math. Inform. 25 (2016), no. 2, 183-196.

[15] Marino, G., Xu H.K.: A general iterative method for nonexpansive mappings in Hilbert spaces, J. Math. Anal. Appl. 318 (2006) 43-52.

[16] Mitrinović, D. S.: Analytic Inequalities. Springer-Verlag, New York (1970).

[17] Moudafi, A.: The split common fixed-point problem for demicontractive mappings, Inverse Problem 26, 587-600 (2010).

[18] Moudafi, A.: A note on the split common fixed-point problem for quasinonexpansive operators, Nonlinear Anal. 74, 4083-4087 (2011).

[19] Pakkaranang, N., Kumam, P., Cholamjiak, P., Suparatulatorn, R., Chaipunya, P., Proximal point algorithms involving fixed point iteration for nonexpansive mappings in $C A T(k)$ spaces. Carpathian J. Math. 34 (2018), no. 2, 229-237.

[20] Qin, X. L., Ansari, Q. H., Yao, J. -C., A forward-backward iterative method for zero points of sum of two accretive operators. Carpathian J. Math. 33 (2017), no. 3, 353-363. 
[21] Țicală, C., Approximating solutions of generalized pseudocontractive variational inequalities by admissible perturbation type iterative methods. Creat. Math. Inform. 22 (2013), no. 2, 237-241.

[22] Tुicală, C., Approximating fixed points of demicontractive mappings by iterative methods defined as admissible perturbations. Creat. Math. Inform. 25 (2016), no. 1, 121-126.

[23] Tुicală, C., Approximating fixed points of asymptotically demicontractive mappings by iterative schemes defined as admissible perturbations. Carpathian J. Math. 33 (2017), no. 3, 381-388.

[24] Wang F.: A new iterative method for the split common fixed point problem in Hilbert spaces, Optimization (2017) DOI: 10.1080/02331934.2016.1274991

[25] Xu, H.K.: Iterative algorithm for nonlinear operators, J. Lond. Math. Soc. 66, 1-17 (2002).

[26] Yang, X. N., Xu, H.-K., Projection algorithms for composite minimization. Carpathian J. Math. 33 (2017), no. 3, 389-397.

[27] Yao, Y., Liou, Y.C., Postolache, M.: Self-adaptive algorithms for the split problem of the demicontractive operators, Optimization, (2017), DOI: $10.1080 / 02331934.2017 .1390747$

[28] Xu H.-K.: Viscosity approximation methods for nonexpansive mappings, J. Math. Anal. Appl. vol. 298, no. 1, pp. 279-291, 2004.

Duangkamon Kitkuan,

KMUTTFixed Point Research Laboratory,

Department of Mathematics,

Room SCL 802 Fixed Point Laboratory,

Science Laboratory Building, Faculty of Science,

King Mongkut's University of Technology Thonburi (KMUTT),

126 Pracha-Uthit Road, Bang Mod, Thrung Khru, Bangkok 10140, Thailand.

Email: or_duangkamon@hotmail.com

Poom Kumam,

KMUTT-Fixed Point Theory and Applications Research Group,

Theoretical and Computational Science Center (TaCS),

Science Laboratory Building, Faculty of Science,

King Mongkut's University of Technology Thonburi (KMUTT),

126 Pracha Uthit Rd., Bang Mod, Thrung Khru,Bangkok 10140, Thailand.

Email: poom.kum@kmutt.ac.th 
Vasile Berinde,

Technical University of Cluj-Napoca,

North University Center of Baia Mare,

Department of Mathematics and Computer Science, Romania.

Academy of Romanian Scientists.

Email: vberinde@cunbm.utcluj.ro

Anantachai Padcharoen,

KMUTTFixed Point Research Laboratory,

Department of Mathematics,

Room SCL 802 Fixed Point Laboratory,

Science Laboratory Building, Faculty of Science,

King Mongkut's University of Technology Thonburi (KMUTT),

126 Pracha-Uthit Road, Bang Mod, Thrung Khru, Bangkok 10140, Thailand.

Email: apadcharoen@yahoo.com 